\title{
Vitrectomy for cystoid macular oedema with attached posterior hyaloid membrane in patients with diabetes
}

\author{
Tomohiro Ikeda, Keiko Sato, Takuya Katano, Yasuhito Hayashi
}

\begin{abstract}
Aim-To report the success of vitrectomy in eliminating cystoid macular oedema and improving vision in three eyes of two patients with diabetic cystoid macular oedema. In all of the eyes there was no ophthalmoscopic evidence of traction from a posterior hyaloid membrane or from proliferative tissue.

Methods-Pars plana vitrectomy was performed on three eyes of two patients with diabetic cystoid macular oedema who did not show traction upon examination with a slit lamp biomicroscope and a scanning laser ophthalmoscope.

Results-Cystoid changes disappeared 1, 3 , and 5 days, postoperatively, and diffuse macular oedema resolved within 2 weeks. The visual acuity was improved and maintained.

Conclusion-Vitrectomy can be effective in some patients with diabetic cystoid macular oedema even in patients who lack evidence of traction by ophthalmoscopy.

(Br F Ophthalmol 1999;83:12-14)
\end{abstract}

Macular photocoagulation is commonly used to treat diabetic patients with macular oedema. ${ }^{1-4}$ Vitrectomy has also been reported to be effective in patients who have a thickened and taut posterior hyaloid membrane exerting traction on the retina. ${ }^{5}$ In such cases, oedema is considered to be mediated by the macular traction from this posterior hyaloid membrane. In cases that lack ophthalmoscopic evidence of traction from such a membrane or from fibrovascular tissue, vitrectomy is not recommended because macular photocoagulation is effective. ${ }^{1-4}$

In Japan, vitrectomy for diffuse macular oedema with an attached posterior hyaloid membrane has been performed at a limited number of institutions even in patients without ophthalmoscopic evidence of traction. This practice is based on the hypothesis that traction of the macula by the posterior hyaloid membrane is present even in patients who do not show an obvious thickened posterior hyaloid membrane. Tachi and Ogino ${ }^{7}$ recently reported the results of vitrectomy for diffuse macular oedema; in their series, a long period was required for the resolution of the oedema in many patients, and it was unclear whether the resolution of oedema was a direct consequence of vitrectomy.

We report the results from three eyes of two patients with diabetic cystoid macular oedema with no evidence of traction by a thickened posterior hyaloid membrane. We shall show that vitrectomy was effective in eliminating the oedema and improving vision.

\section{Patients and methods}

We evaluated three eyes of two patients with diabetic cystoid macular oedema who underwent vitrectomy at Osaka City General Hospital, Osaka, Japan. Detachment of the posterior hyaloid membrane, thickening of the posterior hyaloid membrane, or the presence of an epiretinal or fibrovascular membrane was excluded by preoperative examination with a slit lamp biomicroscope and a scanning laser ophthalmoscope. Informed consent was obtained before surgery.

The surgical technique included pars plana vitrectomy that utilised the standard three port incisions. After core vitrectomy had been performed, the remaining cortical vitreous was aspirated with a flexible tipped cannula or a vitreous cutter to create posterior hyaloid detachment. After confirming the presence of a sheet-like posterior hyaloid membrane, including Weiss's ring, the remaining vitreous was removed.

After surgery, the patients were examined daily during a 2 week hospitalisation and were observed thereafter in the outpatient clinic at intervals of about 2 weeks.

\section{Results}

Intraoperatively, the posterior hyaloid membrane was found to be attached to the macula in all three eyes, and no signs of traction on the macula was noted. There were no intraoperative complications. After the operation, the cystoid changes disappeared by 1,3 , and 5 days, and the diffuse macular oedema resolved within 2 weeks. The following two cases illustrate the course of the changes in the three eyes.

CASE 1

This patient was a 41 year old male with a 10 year history of NIDDM. He had undergone panretinal photocoagulation for proliferative diabetic retinopathy 6 months before his first visit to our institution. At the initial examination, his corrected visual acuity was 0.5 in the right eye and 0.06 in the left eye. Cystoid macular oedema was observed in both eyes. Vitrectomy was performed first on the left eye. Disc neovascularisation was noted preoperatively, but no macular traction was observed. The cystoid changes disappeared unexpectedly
Accepted for publication 12 May 1998 



Figure 1 Case 1, left eye. (A) Late phase fluorescein angiogram obtained preoperatively demonstrating cystoid macular oedema. Marked hyperfluorescence appears honeycombed in the outer portions of the macula and stellate in the centre of the macula. Neovascularisation on the disc and retina is also evident. (B) Arteriovenous phase of fluorescein angiogram taken 4 months after vitrectomy. There are retinal pigment epithelial window defects in the centre of the macula and in the surrounding area. (C) Late phase fluorescein angiogram shows hyperfluorescence due to window defect. Macular leakage and pooling of fluorescein in the cystoid spaces has been markedly reduced.

3 days after surgery, and the diffuse oedema was also reabsorbed within 9 days. Figure $1 \mathrm{~A}$ shows a preoperative fluorescein angiogram, and Figures $1 \mathrm{~B}$ and $\mathrm{C}$ show those taken 4 months after surgery. Cystoid macular oedema and macular leakage were markedly reduced.

Subsequently, the visual acuity in the right eye decreased gradually to a corrected acuity of 0.2 . Vitrectomy was then performed on this eye. Expecting a favourable effect similar to that in the left eye, we prospectively made closer observation on the course of the retinal changes. Because of the good results on the left eye, a careful search was made preoperatively and intraoperatively for traction on the macula by the hyaloid membrane or fibrous tissues and none was found. Cystoid changes disappeared 5 days after vitrectomy and diffuse oedema
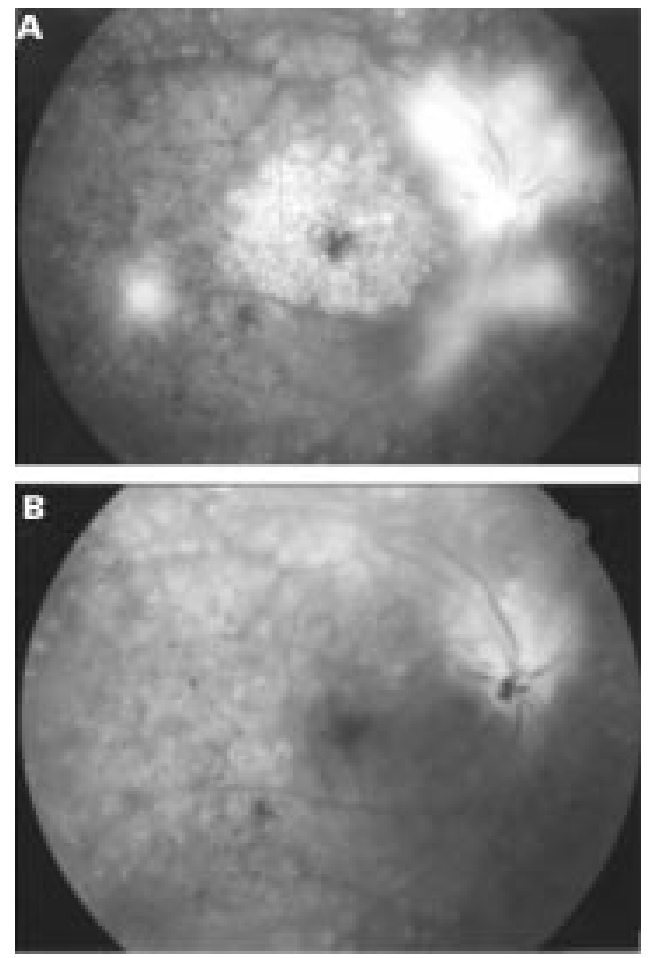

Figure 2 Case 1, right eye. (A) Late phase fluorescein angiogram obtained preoperatively shows cystoid macular oedema. Neovascularisation on the disc and retina are also evident. (B) Late phase fuorescein angiogram taken 1 week after vitrectomy demonstrating that the diffuse macular leakage is markedly reduced, and the pooling of fluorescein in the cystoid spaces has disappeared.

resolved 12 days after vitrectomy. Cystoid changes were not detected by fluorescein angiography performed 1 week after vitrectomy (Fig 2B). Corrected visual acuity was 0.7 in the right eye 20 months after vitrectomy, and 0.1 in the left eye 24 months after vitrectomy. Macular oedema had resolved completely in both eyes. The postoperative vision was poor in the left eye because of the marked atrophy of the pigment epithelium that was observed before surgery.

CASE 2

This patient was a 25 year old female with a 21 year history of IDDM. At the initial examination, the corrected visual acuity was 1.2 in both eyes. The diabetic retinopathy at the initial examination was at a non-proliferative stage but panretinal photocoagulation was performed because retinopathy had become proliferative at a later examination. Cystoid macular oedema developed in the right and left eyes at 2 and 6 months, respectively, after photocoagulation. Corrected visual acuity was 1.2 in both eyes at the onset of cystoid macular oedema. However, it decreased gradually in the right eye and when it became $0.4,18$ months after the onset of oedema, vitrectomy was performed. Disc neovascularisation was observed (Fig 3A), but no fibrous tissue was noted in the posterior vitreous. Careful examination did not reveal any traction on the macula.

Cystoid changes disappeared the day after vitrectomy, and the foveal reflex was confirmed. Evidence of cystoid macular oedema was absent from fluorescein angiograms made 

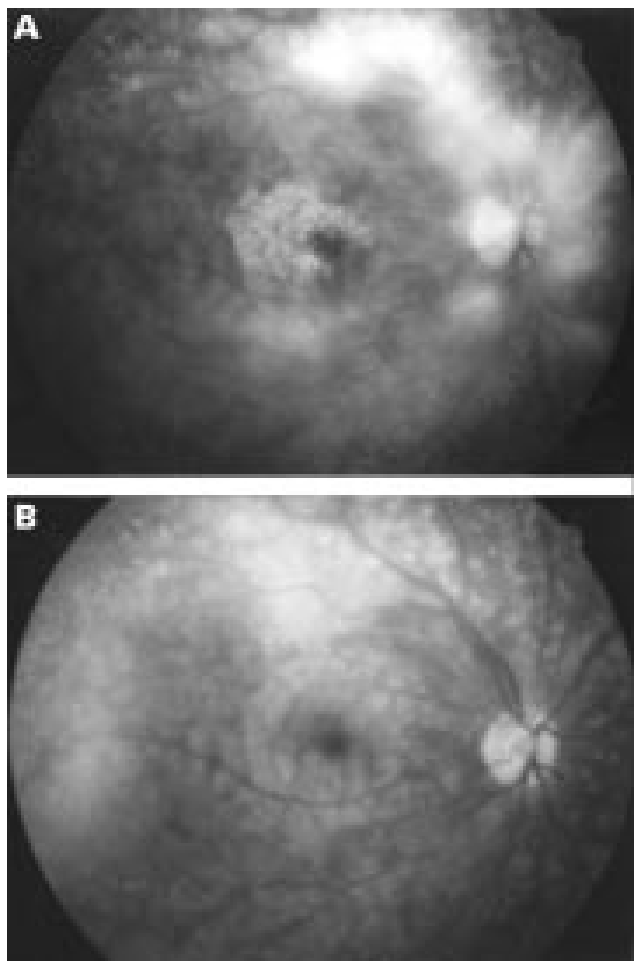

Figure 3 Case 2. (A) Preoperative late phase fluorescein angiogram demonstrates cystoid macular oedema. Marked leakage from the disc neovascularisation is also evident. (B) Late phase fluorescein angiogram taken 1 week after vitrectomy shows a resolution of cystoid macular oedema. Newly formed vessels on the disc have been completely eliminated.

1 week after surgery (Fig 3B), and corrected visual acuity had recovered to 1.0 after 2 weeks. Corrected visual acuity is maintained at 1.015 months after surgery. The left eye is being observed without further treatment, because the corrected visual acuity is 1.0 despite the presence of cystoid macular oedema.

\section{Discussion}

Our results suggest that vitrectomy is effective in some patients who lacked ophthalmoscopic evidence of traction from the posterior hyaloid membrane or proliferative tissue. The rapid disappearance of the cystoid changes and diffuse oedema after vitrectomy clearly indicated an involvement of the vitreous in the occurrence of oedema.

Although the mechanism of the oedema in such patients is not clear, traction by the posterior hyaloid membrane seems to be the most likely cause, as it is in cases having a visible thickened posterior hyaloid membrane. ${ }^{56}$ In these two patients, macular oedema gradually worsened after panretinal photocoagulation, which probably altered the posterior hyaloid membrane causing it to exert tangential traction $^{8}$ on the macula. If traction from the posterior hyaloid membrane is the primary cause of the oedema, the effectiveness of macular photocoagulation in such cases is questionable. Because photocoagulation can result in a decrease in retinal sensitivity ${ }^{9}$ and in visual loss due to a progressive enlargement of the laser scars ${ }^{10}{ }^{11}$ or subretinal fibrosis, ${ }^{12}$ it is not necessarily a safe treatment. Although vitrectomy also occasionally causes severe complications, ${ }^{13}$ the complications from simple vitrectomy has been reduced by recent improvements in surgical technique and instrumentation. Because vitrectomy, unlike photocoagulation, does not cause irreversible retinochoroidal impairment, the procedure, if it is effective, should be included among the alternative treatments for patients with diabetic macular oedema.

As demonstrated in our study, vitrectomy can result in resolution of the macular oedema in some patients with an attached posterior hyaloid membrane. Multicentre, randomised, controlled studies of the effectiveness of vitrectomy for macular oedema compared with photocoagulation, are needed. In the meantime, we recommend that vitrectomy be considered for the treatment of cystoid macular oedema even in the absence of a thickened posterior hyaloid membrane.

1 British Multicentre Study Group. Photocoagulation in treatment of diabetic maculopathy. Lancet 1975;2:111013.

2 British Multicentre Study Group. Photocoagulation for diabetic maculopathy. Diabetes 1983;32:1010-6.

3 Early Treatment Diabetic Retinopathy Study Research Group. Photocoagulation for diabetic macular edema: Early Treatment Diabetic Retinopathy Study report number 1. Arch Ophthalmol 1985;103:1796-806.

4 Early Treatment Diabetic Retinopathy Study Research Group. Photocoagulation for diabetic macular edema: Early Treatment Diabetic Retinopathy Study report number 4. Int Ophthalmol Clin 1987;27:265-72.

5 Lewis H, Abrams GW, Blumenkranz MS, et al. Vitrectomy for diabetic macular traction and edema associated with posterior hyaloidal traction. Ophthalmology 1992;99:753-9.

6 Harbour JW, Smiddy WE, Flynn HW Jr, et al. Vitrectomy for diabetic macular edema associated with a thickened and taut posterior hyaloid membrane. Am $\mathcal{f}$ Ophthalmol 1996;121:405-13.

7 Tachi N, Ogino N. Vitrectomy for diffuse macular edema in cases of diabetic retinopathy. Am f Ophthalmol 1996;122: 258-60

8 Kishi S, Shimizu K. Clinical manifestations of posterior precortical vitreous pocket in proliferative diabetic retinopathy. Ophthalmology 1993;100:225-9.

9 Striph GG, Hart WM, Olk RJ. Modified grid laser photocoagulation for diabetic macular edema. The effect on the central visual field. Ophthalmology 1988;95:1673-9.

10 Schatz H, Madeira D, McDonald HR, et al. Progressive enlargement of laser scars following grid laser photocoagu-
lation for diffuse diabetic macular edema. Arch Ophthalmol 1991;109:1549-51.

11 Rutledge BK, Wallow IH, Poulsen GL. Sub-pigment epithelial membranes after photocoagulation for diabetic macular edema. Arch Ophthalmol 1993;111:608-13.

12 Guyer DR, D'Amico DJ, Smith CW. Subretinal fibrosis after laser photocoagulation for diabetic macular edema. Am $\mathcal{F}$ Ophthalmol 1992;113:652-6.

13 Peyman GA, Schulman JA. Intravitreal surgery. 2nd ed. Connecticut: Appleton and Lange, 1994:813-50. 